\title{
Structural, magnetic, and spin dynamical properties of the polar antiferromagnets $\mathrm{Ni}_{3-x} \mathrm{Co}_{x} \mathrm{TeO}_{6}(x=1,2)$
}

\author{
Stella Skiadopoulou $\odot,{ }^{1,2,3, *}$ Maria Retuerto, ${ }^{4}$ Fedir Borodavka $\odot,{ }^{1}$ Christelle Kadlec, ${ }^{1}$ Filip Kadlec $\odot,{ }^{1}$ Martin Míšek, \\ Jan Prokleška, ${ }^{5}$ Zheng Deng, ${ }^{4}$ Xiaoyan Tan, ${ }^{4, \dagger}$ Corey Frank $\odot,{ }^{4}$ Jose A. Alonso $\odot,{ }^{6}$ Maria Tereza Fernandez-Diaz, ${ }^{7}$ \\ Mark Croft, ${ }^{8}$ Fabio Orlandi, ${ }^{9}$ Pascal Manuel, ${ }^{9}$ Emma McCabe $\odot,{ }^{10}$ Dominik Legut, ${ }^{2}$ \\ Martha Greenblatt, ${ }^{4}$ and Stanislav Kamba ${ }^{1}{ }^{1}$ \\ ${ }^{1}$ Institute of Physics, Czech Academy of Sciences, Na Slovance 2, 18221 Prague 8, Czech Republic \\ ${ }^{2}$ IT4Innovations, VSB Technical University of Ostrava, 17, Listopadu 2172/15, CZ-708 00 Ostrava-Poruba, Czech Republic \\ ${ }^{3}$ School of Physics, CRANN, Trinity College, Dublin 2, Ireland \\ ${ }^{4}$ Department of Chemistry and Chemical Biology, Rutgers, The State University of New Jersey, 610 Taylor Road, Piscataway, \\ New Jersey 08854, USA \\ ${ }^{5}$ Department of Condensed Matter Physics, Faculty of Mathematics and Physics, Charles University, Ke Karlovu 5 , \\ 12116 Prague 2, Czech Republic \\ ${ }^{6}$ Instituto de Ciencia de Materiales de Madrid, C.S.I.C., Cantoblanco, E-28049 Madrid, Spain \\ ${ }^{7}$ Institut Laue Langevin, BP 156X, Grenoble, F-38042, France \\ ${ }^{8}$ Department of Physics and Astronomy, Rutgers, the State University of New Jersey, 136 Frelinghusen Road, Piscataway, \\ New Jersey 08854, USA \\ ${ }^{9}$ ISIS Facility, STFC, Rutherford Appleton Laboratory, Chilton, Didcot, Oxfordshire, OX11 OQX, United Kingdom \\ ${ }^{10}$ School of Physical Sciences, University of Kent, Canterbury, Kent, CT2 7NH, United Kingdom
}

(Received 10 September 2019; published 21 January 2020)

\begin{abstract}
We present results of a multimethod investigation of the polar antiferromagnets $\mathrm{Ni}_{2} \mathrm{CoTeO}_{6}$ and $\mathrm{NiCo}_{2} \mathrm{TeO}_{6}$, inspired by the colossal magnetoelectric effect present in $\mathrm{Ni}_{3} \mathrm{TeO}_{6}$. Both compounds crystalize in the same polar space group $R 3$ as $\mathrm{Ni}_{3} \mathrm{TeO}_{6}$, preserving the crystal symmetry at least from room temperature down to $2 \mathrm{~K} \mathrm{Ni}_{2} \mathrm{CoTeO}_{6}$ and $\mathrm{NiCo}_{2} \mathrm{TeO}_{6}$ undergo antiferromagnetic phase transitions at $T_{\mathrm{N}}=55$ and $52 \mathrm{~K}$, and spin-flop transitions at an external magnetic field of approximately 8 and $4 \mathrm{~T}$, respectively. Both compounds present an incommensurate antiferromagnetic helical structure with spins lying in the $a b$ plane, in contrast to the collinear one along the $c$ axis in $\mathrm{Ni}_{3} \mathrm{TeO}_{6}$. Moreover, dielectric anomalies are observed at their antiferromagnetic phase transitions, suggesting a magnetoelectric behavior. Spin and lattice dynamics studies by a combination of infrared, Raman, and terahertz spectroscopies were performed. Below $T_{\mathrm{N}}$, in both $\mathrm{Ni}_{2} \mathrm{CoTeO}_{6}$ and $\mathrm{NiCo}_{2} \mathrm{TeO}_{6}$, low-frequency spin excitations extremely sensitive to external magnetic field were observed. At least one of these magnons was simultaneously seen in Raman and $\mathrm{THz}$ spectra of both compounds, therefore we propose to assign them to electromagnons.
\end{abstract}

DOI: 10.1103/PhysRevB.101.014429

\section{INTRODUCTION}

The polar antiferromagnet $\mathrm{Ni}_{3} \mathrm{TeO}_{6}$ exhibits static (colossal) [1] and dynamical [2] magnetoelectric (ME) effects. A collinear antiferromagnetic (AFM) order appears below the Néel temperature $T_{\mathrm{N}}=53 \mathrm{~K}$ [3], giving rise to spin-induced polarization of up to $3280 \mu \mathrm{C} / \mathrm{m}^{2}$ (at $2 \mathrm{~K}$ ) [1]. Two spin excitations observed simultaneously in Raman and time-domain $\mathrm{THz}$ spectra emerge below $T_{\mathrm{N}}$ [2]. These magnons show high tunability in external magnetic field. Due to symmetry considerations, these excitations are assigned to electromagnons, i.e., electrically active magnons $[2,4,5]$. Since $\mathrm{Ni}_{3} \mathrm{TeO}_{6}$ presents a collinear AFM spin structure, the spinlattice coupling mechanism (symmetric exchange striction) is

\footnotetext{
*Corresponding author: skiadops@tcd.ie
}

$\dagger$ Present address: Department of Chemistry and Biochemistry, George Mason University, 4400 University Dr, Fairfax, Virginia 22030, USA. most probably responsible for the emergent dynamical ME effect. A similar polar antiferromagnet $\mathrm{Ni}_{2} \mathrm{MnTeO}_{6}$, undergoes an AFM phase transition at $T_{\mathrm{N}} \approx 70 \mathrm{~K}$, almost $20 \mathrm{~K}$ higher than $\mathrm{Ni}_{3} \mathrm{TeO}_{6}$, while keeping the same polar rhombohedral $R 3$ space group symmetry [6]. A dielectric anomaly at $T_{\mathrm{N}}$ suggests a ME behavior, and a magnetic field-sensitive spin excitation appears below $T_{\mathrm{N}}$ in the $\mathrm{THz}$ range.

The cryolite-related $\mathrm{Co}_{3} \mathrm{TeO}_{6}$ with the monoclinic $C 2 / c$ space group symmetry down to $1.9 \mathrm{~K}$ [7] undergoes at least three magnetic phase transitions within the range from 26 to $16 \mathrm{~K}$ [7-13]. Two main competing reports on the complex magnetic ordering in $\mathrm{Co}_{3} \mathrm{TeO}_{6}$ suggest either switching from an incommensurate collinear AFM $(\sim 26 \mathrm{~K})$ to a commensurate noncollinear AFM structure $(\sim 17 \mathrm{~K})$ with multiple propagation vectors $[7,10]$, or a coexistence of two layers with commensurate collinear and incommensurate noncollinear AFM spin orderings [12]. In the lowest temperature phase, a magnetically induced polarization of up to $160 \mu \mathrm{C} / \mathrm{m}^{2}(P \| c$, $H \| a, 5 \mathrm{~K}, 9 \mathrm{~T})$ emerges [7,8], possibly via the spin-current mechanism (antisymmetric exchange) [11]. 
The peculiar magnetoelectricity and complex magnetic ordering of $\mathrm{Co}_{3} \mathrm{TeO}_{6}$, in combination with the colossal magnetoelectric coupling in $\mathrm{Ni}_{3} \mathrm{TeO}_{6}$, led to the concept of $\mathrm{Ni}$ substitution by $\mathrm{Co}$ in $\mathrm{Ni}_{3} \mathrm{TeO}_{6}$. For the current study, $\mathrm{Ni}_{2} \mathrm{CoTeO}_{6}$ and $\mathrm{NiCo}_{2} \mathrm{TeO}_{6}$ were synthesized in the form of single crystals and polycrystalline samples. Partial Ni substitution by Co preserves the polar $R 3$ crystal symmetry of $\mathrm{Ni}_{3} \mathrm{TeO}_{6}$, while inducing a noncollinear helical spin structure, in contrast to the collinear cases of $\mathrm{Ni}_{3} \mathrm{TeO}_{6}$ and $\mathrm{Ni}_{2} \mathrm{MnTeO}_{6}$. The helical spin configurations in $\mathrm{Ni}_{2} \mathrm{CoTeO}_{6}$ and $\mathrm{NiCo}_{2} \mathrm{TeO}_{6}$ yield hysteretic spin-flop transitions; in comparison, spin-flop transitions in the collinear cases are nonhysteretic. In this article, we discuss spin and lattice excitations with an emphasis on the magneticfield sensitive magnons in the $\mathrm{THz}$ range.

\section{EXPERIMENTAL SECTION}

Polycrystalline $\mathrm{Ni}_{2} \mathrm{CoTeO}_{6}$ and $\mathrm{NiCo}_{2} \mathrm{TeO}_{6}$ were prepared by solid state reactions. Stoichiometric amounts of $\mathrm{NiO}$, $\mathrm{TeO}_{2}$, and $\mathrm{MnCO}_{3}$ were mixed, ground together, and heated in a tubular furnace under oxygen flux at $800{ }^{\circ} \mathrm{C}$ with intermediate grindings between 700 and $800{ }^{\circ} \mathrm{C}$.

Single crystals of both materials were prepared by the flux method by mixing the corresponding mixed oxide $\left(\mathrm{Ni}_{2} \mathrm{CoTeO}_{6}\right.$ or $\left.\mathrm{NiCo}_{2} \mathrm{TeO}_{6}\right)$ with $\mathrm{V}_{2} \mathrm{O}_{5}, \mathrm{TeO}_{2}, \mathrm{NaCl}$, and $\mathrm{KCl}$ in a molar ratio of the five compounds of 1:5:10:10:5. The mixture was heated to $830{ }^{\circ} \mathrm{C}$ and kept at this temperature during three days. The crystals were cooled to $600{ }^{\circ} \mathrm{C}$ during five days. Plate-shaped crystals were obtained with lateral dimensions of $\sim 2 \mathrm{~mm}$ for $\mathrm{Ni}_{2} \mathrm{CoTeO}_{6}$ and less than $1 \mathrm{~mm}$ for $\mathrm{NiCo}_{2} \mathrm{TeO}_{6}$. (See Fig. S1.1 in the Supplemental Material (SM) [14], see also Refs. [15-19] therein).

The samples were initially characterized by powder x-ray diffraction (XRD) $\left(\mathrm{Cu} \mathrm{K}_{\alpha}=1.5406 \AA\right)$. The structure of the single crystals was determined by single-crystal $\mathrm{x}$-ray data collected on a Bruker Smart APEX CCD diffractometer with graphite-monochromatized $\operatorname{Mo~}_{\alpha}$ radiation $(\lambda=0.71073 \AA)$ at $100 \mathrm{~K}$.

Time-of-flight neutron powder diffraction (NPD) data were collected for $\mathrm{NiCo}_{2} \mathrm{TeO}_{6}$ on the WISH diffractometer (target station 2) at the ISIS neutron and muon source [20]; 250 mg of powder were placed in a thin-walled, cylindrical vanadium can and cooled to $1.5 \mathrm{~K}$. High quality data sets with angular steps equivalent to $40 \mu \AA$ in direct space were collected at 1.5 , 32 ,and $60 \mathrm{~K}$. Shorter scans with finer steps of $6.7 \mu \AA$ were collected at intermediate temperatures, and the data at $100 \mathrm{~K}$ were obtained with $16.8 \mu \AA$ steps. Constant wavelength NPD data were collected for $\mathrm{Ni}_{2} \mathrm{CoTeO}_{6} ; 250 \mathrm{mg}$ of powder were placed in a 6- mm-diameter vanadium can. High resolution scans were collected on the D2B diffractometer $(\lambda \approx 1.59 \AA)$ at 1.5 and $300 \mathrm{~K}$. Variable temperature data were also collected on the high flux diffractometer D20 $(\lambda \approx 2.41 \AA)$ to follow the magnetic ordering.

TOPASACADEMIC [21] software was used for initial refinements using data collected in the paramagnetic region to determine the Ni/Co distribution over $3 a$ sites. The cation distribution was then fixed for refinements using Jana2006 [22] using data collected at base temperature and on warming. Group theory calculations and magnetic symmetry analysis were performed with the help of the ISODISTORT and ISOTROPY software [23,24].

Radio-frequency dielectric measurements were performed using a NOVOCONTROL $\alpha$-AN impedance analyzer in conjunction with a JANIS ST-100 cryostat.

Near-normal incidence infrared (IR) reflectivity spectra of $\mathrm{Ni}_{3-x} \mathrm{Co}_{x} \mathrm{TeO}_{6}(x=1,2)$ ceramics were measured using a Fourier-transform IR spectrometer Bruker IFS $113 \mathrm{v}$ in the frequency range of $20-3000 \mathrm{~cm}^{-1}(0.6-90 \mathrm{THz})$ at room temperature; the low-temperature spectral range was reduced to $20-650 \mathrm{~cm}^{-1}$ due to the limited transparency of the cryostat windows from polyethylene. A pyroelectric deuterated triglycine sulfate detector was used for the room-temperature measurements, whereas a He-cooled (operating temperature 1.6 K) Si bolometer was used for the low-temperature measurements down to $7 \mathrm{~K}$.

Time-domain terahertz experiments were conducted in the spectral range from 3 to $60 \mathrm{~cm}^{-1}(0.09-1.8 \mathrm{THz})$, performed in the transmission geometry with a custom-made time-domain terahertz spectrometer. A train of femtosecond pulses was produced by a femtosecond Ti:sapphire laser oscillator (Coherent, Mira), which generated linearly polarized broadband $\mathrm{THz}$ pulses radiated by a photoconducting switch TeraSED (Giga-Optics). A gated detection scheme based on electro-optic sampling with a 1-mm-thick [110] ZnTe crystal as a sensor allowed us to measure the time profile of the electric field of the transmitted $\mathrm{THz}$ pulse. For low-temperature $\mathrm{THz}$ and IR measurements, two Oxford Instruments Optistat cryostats with mylar and polyethylene windows, respectively, were used. External magnetic field $\mathrm{THz}$ experiments with $\mu_{\mathrm{o}} H_{\mathrm{ext}} \leqslant 7 \mathrm{~T}$ were conducted with an Oxford Instruments Spectromag cryostat in the Voigt configuration (with both $\boldsymbol{H}_{\text {ext }} \| \boldsymbol{E}^{\omega}$ and $\boldsymbol{H}_{\text {ext }} \perp \boldsymbol{E}^{\omega}$ ), where $\boldsymbol{E}^{\omega}$ denotes the electrical component of the electromagnetic radiation.

The IR reflectivity $R(\omega)$ and $\mathrm{THz}$ complex permittivity spectra $\varepsilon(\omega)$ were fitted assuming a sum of $N$ independent damped harmonic oscillators, expressed as [25]

$$
\varepsilon(\omega)=\varepsilon_{\infty}+\sum_{j=1}^{N} \frac{\Delta \varepsilon_{j} \omega_{\mathrm{TO} j}^{2}}{\omega_{\mathrm{TO}_{j}}^{2}-\omega^{2}+i \omega \gamma_{\mathrm{TO} j}},
$$

where $\Delta \varepsilon_{j}$ is the dielectric strength of the $j$ th mode, $\omega_{\mathrm{TO} j}$ are the frequencies of the $j$ th transverse optical (TO) phonons, and $\gamma_{\mathrm{TO} j}$ are the corresponding damping constants. $\varepsilon_{\infty}$ is the high-frequency (electronic) contribution to the permittivity, determined from the room-temperature frequencyindependent reflectivity tail above the phonon frequencies. The complex magnetic permeability $\mu$ may be expressed in a similar way, and then the reflectivity, the complex dielectric function and the complex magnetic permeability are related by

$$
R(\omega)=\left|\frac{\sqrt{\varepsilon(\omega)}-1}{\sqrt{\varepsilon(\omega)}+1}\right|^{2}, \quad R(\omega)=\left|\frac{\sqrt{\varepsilon}-\sqrt{\mu}}{\sqrt{\varepsilon}+\sqrt{\mu}}\right|^{2} .
$$

Raman studies of single crystals were performed with a Renishaw RM 1000 Micro-Raman spectrometer with Bragg filters, equipped with an Oxford Instruments Microstat continuous-flow optical He cryostat. The experiments were conducted in the backscattering geometry in the 
$5-1800 \mathrm{~cm}^{-1}$ range. An $\mathrm{Ar}^{+}$-ion laser operating at $514.5 \mathrm{~nm}$ was used. The spectra were carefully fit with a sum of independent damped harmonic oscillators multiplied by the corresponding Stokes temperature factor [26].

\section{RESULTS AND DISCUSSION}

\section{A. Structural characterization}

Single crystals and polycrystalline samples of $\mathrm{Ni}_{2} \mathrm{CoTeO}_{6}$ and $\mathrm{NiCO}_{2} \mathrm{TeO}_{6}$ were characterized by XRD and NPD, revealing pure phases with the noncentrosymmetric $R 3$ space group symmetry. The XRD and NPD patterns, unit cell parameters, atomic positions, and bond distances, are presented in Secs. S2 and S3 in the SM [14]. Both compounds present layers of edge-sharing $\mathrm{NiO}_{6}, \mathrm{CoO}_{6}$, and $\mathrm{TeO}_{6}$ octahedra, where the central $\mathrm{Ni}, \mathrm{Co}$, and Te ions are slightly (by $\sim 0.1 \AA$ ) displaced from the centers of the oxygen octahedra cages.

\section{B. Magnetic structures}

The magnetic structures of both compounds, determined by NPD, correspond to an incommensurate AFM helical with the magnetic moments lying in the $a b$ plane, in clear contrast with the collinear out-of-plane spin structure of $\mathrm{Ni}_{3} \mathrm{TeO}_{6}$ and $\mathrm{Ni}_{2} \mathrm{MnTeO}_{6}[1,3,6]$. The magnetic propagation vectors are $\boldsymbol{k}_{1}=(0,0,1.299(4))$ and $\boldsymbol{k}_{2}=(0,0,1.2109(1))$, for $\mathrm{Ni}_{2} \mathrm{CoTeO}_{6}$ and $\mathrm{NiCo}_{2} \mathrm{TeO}_{6}$ at 3 and $1.5 \mathrm{~K}$, respectively. In both compounds the magnetic structure is described by the superspace group $R 31^{\prime}(O 0 \gamma) t s$, corresponding to the $\mathrm{m} \Lambda \mathrm{LE} 2$ irreducible representation. The helical structure is characterized by ferromagnetic (FM) $a b$ layers rotating along the $c$ axis (Fig. 1) yielding an AFM structure. It is interesting to note that the sole difference between the two compounds is the periodicity of the helix along $c$, which increases with

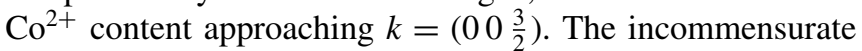
nature of the magnetic structures suggests some frustration in magnetic exchanges along the $c$ axis and a lack of a clear anisotropy direction in the $a b$ plane. Details of the structure refinements, Rietveld plots, values of the magnetic moments as well as the temperature evolutions of the magnetic structures are reported in Sec. S3 in the Supplemental Material [14].

Note that the related materials $\mathrm{Ni}_{2-x} \mathrm{Co}_{x} \mathrm{ScSbO}_{6}(x=$ $0-1.5)$ and $\mathrm{Mn}_{2} \mathrm{InSbO}_{6}$ have similar polar $R 3$ crystal structures, but their magnetic structures differ substantially. The helical spin structure of $\mathrm{Ni}_{2} \mathrm{ScSbO}_{6}$ is incommensurately modulated with an in-plane propagation vector $\boldsymbol{k}=$ [0 0.036(1) 0], corresponding to an almost 40 times longer helix than that in the $\mathrm{Ni}_{3-x} \mathrm{Co}_{x} \mathrm{TeO}_{6}$ compounds [27]. With the addition of $\mathrm{Co}$, the $\mathrm{Ni}_{2-x} \mathrm{Co}_{x} \mathrm{ScSbO}_{6}(x=0.5-1.5)$ compounds present a series of long-periodicity commensurate helices with $\boldsymbol{k}=[001 / 3 n]$, where $n=5,6,8$ and 10 for $x=0.5,1,1.5$, and 2 , thus the helix length increases with increasing Co contents [28].

Finally, the double-corundum compound $\mathrm{Mn}_{2} \mathrm{InSbO}_{6}$ exhibits three magnetic phase transitions below $38 \mathrm{~K}$, and the structure may exhibit a commensurately modulated helix with a propagation vector $\boldsymbol{k}=[000]$, an incommensurate one with $\boldsymbol{k}=\left[\begin{array}{ll}0 & 0 k_{\mathrm{z}}\end{array}\right]$, or a commensurate one with $k_{\mathrm{z}}=1 / 8[29]$.

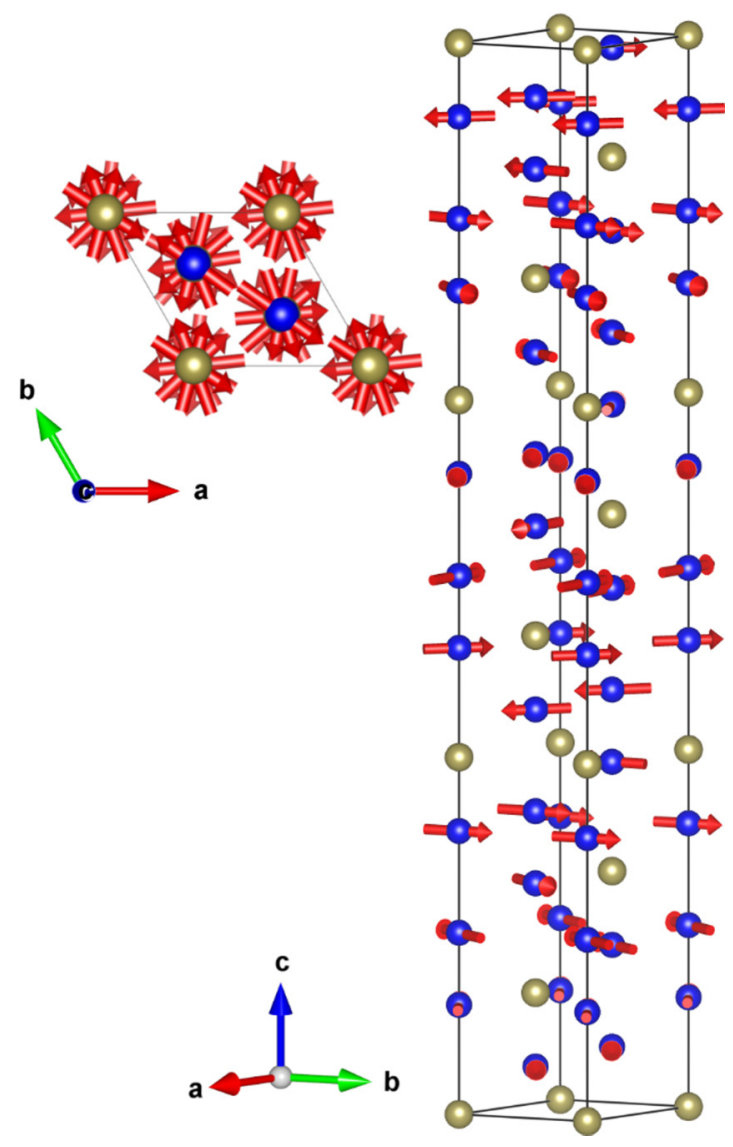

FIG. 1. Schematic representation of the incommensurate helical magnetic structure of $\mathrm{Ni}_{2} \mathrm{CoTeO}_{6}$ and $\mathrm{NiCo}_{2} \mathrm{TeO}_{6}$ (the magnetic $\mathrm{Ni} / \mathrm{Co}$ sites are presented as blue spheres, whereas $\mathrm{Te}$ atoms are shown in gold, oxygen atoms are not shown for clarity). The magnetic structures of both compounds are quite similar, with a small difference between the magnetic propagation vectors: $k_{1}=$ $(0,0,1.299(4))$ and $k_{2}=(0,0,1.2109(1))$, for $\mathrm{Ni}_{2} \mathrm{CoTeO}_{6}$ and $\mathrm{NiCo}_{2} \mathrm{TeO}_{6}$, respectively.

\section{Magnetic and dielectric properties}

The temperature dependences of the magnetization in $\mathrm{Ni}_{2} \mathrm{CoTeO}_{6}$ and $\mathrm{NiCo}_{2} \mathrm{TeO}_{6}$ polycrystalline samples reveal AFM phase transitions at 54 and $49 \mathrm{~K}$, respectively [Fig. 2(a)]. Similarly, the single crystals of $\mathrm{Ni}_{2} \mathrm{CoTeO}_{6}$ and $\mathrm{NiCo}_{2} \mathrm{TeO}_{6}$ exhibit AFM orderings at slightly higher temperatures of 55 and $52 \mathrm{~K}$, respectively [Figs. S2(a) and S2(b) in the SM) [14]. Here we chose to show the results of the ceramic samples, in order to provide a comparison with the dielectric measurements done exclusively on ceramics.

The magnetic transitions in both samples resemble that of $\mathrm{Ni}_{3} \mathrm{TeO}_{6}$, unlike $\mathrm{Ni}_{2} \mathrm{MnTeO}_{6}$, which orders antiferromagnetically at around $70 \mathrm{~K}$ [6]. The single crystals were measured after applying $0.1 \mathrm{~T}$, at zero field cooling, and for two different magnetic field configurations: $\boldsymbol{H}_{\text {ext }} \| c$ axis and $\boldsymbol{H}_{\text {ext }} \| a b$ plane. In contrast to $\mathrm{Ni}_{2} \mathrm{MnTeO}_{6}$ exhibiting a $c$-easy-axis magnetization, both Co compounds present $a b$-easy-plane magnetization, confirming the magnetic structure indicated by the NPD experiments. An additional anomaly at around $35 \mathrm{~K}$ is present in the $\mathrm{Ni}_{2} \mathrm{CoTeO}_{6}$ single crystal [Fig. S5(a) in the SM] [14], possibly due to another magnetic phase transition. 

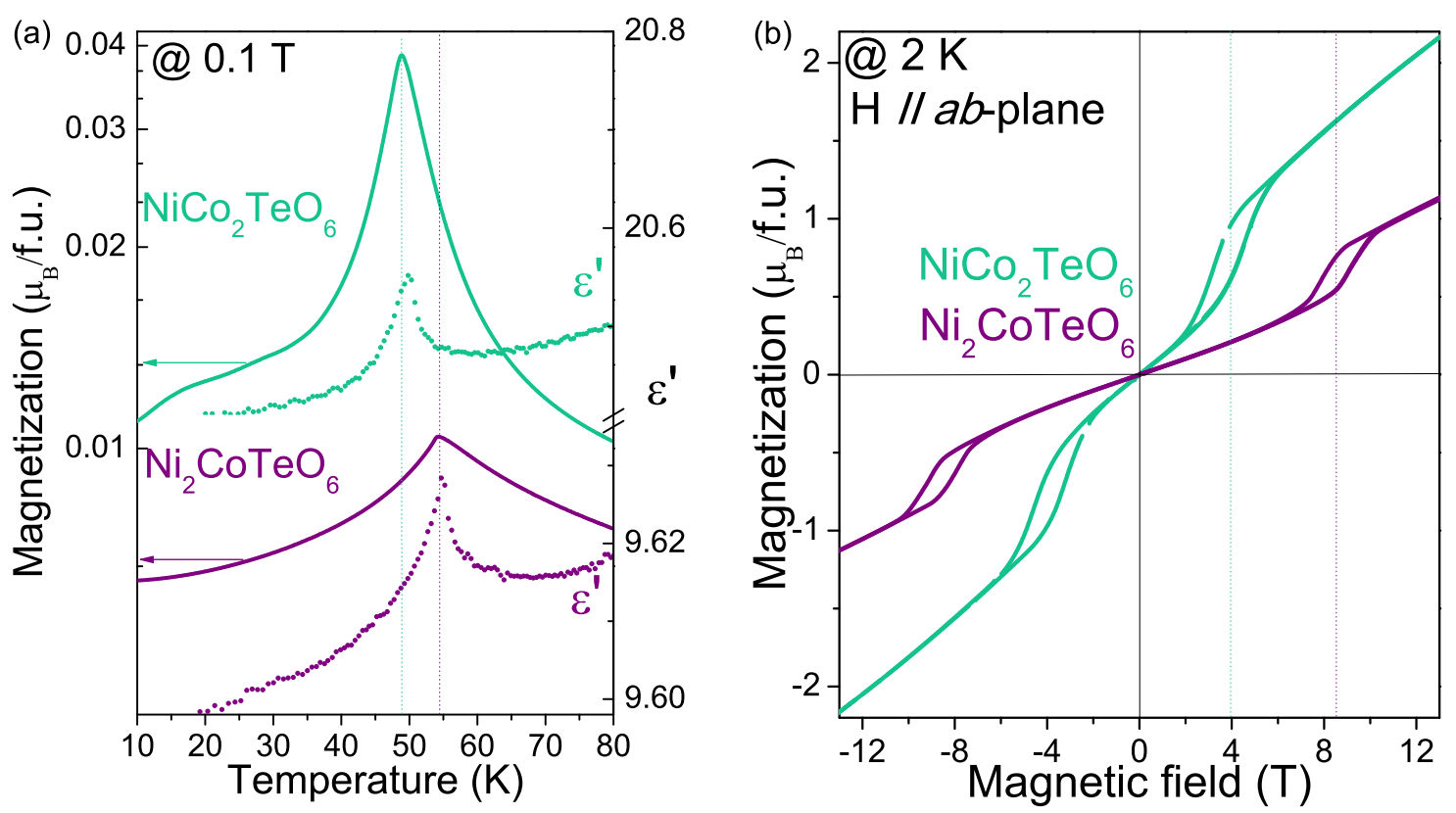

FIG. 2. (a) Temperature dependences of magnetization (at $0.1 \mathrm{~T}$; logarithmic scale) and permittivity (at $0.1 \mathrm{MHz}^{2}$ for $\mathrm{Ni}_{2} \mathrm{CoTeO}_{6}$ and $\mathrm{NiCo}_{2} \mathrm{TeO}_{6}$ ceramics, presenting AFM phase transition at 54 and $49 \mathrm{~K}$, respectively. The dielectric anomalies also appear at the same temperatures for each compound. (b) Magnetic field dependences of magnetization with $H_{\text {ext }} \| a b$ plane at $2 \mathrm{~K}_{\text {for }} \mathrm{Ni}_{2} \mathrm{CoTeO}_{6}$ and $\mathrm{NiCo}_{2} \mathrm{TeO}_{6}$ single crystals, revealing spin flop transitions at approximately 8 and $4 \mathrm{~T}$, respectively.

The same anomaly is absent in the ceramic samples (neither was it observed in NPD), due to random orientations of the grains. As anticipated due to the multiferroic behavior of $\mathrm{Ni}_{3} \mathrm{TeO}_{6}$ and $\mathrm{Ni}_{2} \mathrm{MnTeO}_{6}$, also $\mathrm{Ni}_{2} \mathrm{CoTeO}_{6}$ and $\mathrm{NiCo}_{2} \mathrm{TeO}_{6}$, there is evidence of an interplay of magnetic and dielectric properties, as indicated by the dielectric anomaly seen at $T_{\mathrm{N}}$ [Fig. 2 (a)]. The critical temperatures amount to 53 and $47 \mathrm{~K}$ for $\mathrm{Ni}_{2} \mathrm{CoTeO}_{6}$ and $\mathrm{NiCo}_{2} \mathrm{TeO}_{6}$ ceramic samples, respectively, slightly lower $(\sim 1-2 \mathrm{~K})$ than the magnetic anomalies. Such a discrepancy, which lies close to the experimental error, may also originate in thermo-lag effect at the sample-thermocouple contact during the measurements. The permittivity values of the two compounds differ, with $\mathrm{Ni}_{2} \mathrm{CoTeO}_{6}$ close to $\mathrm{Ni}_{3} \mathrm{TeO}_{6}$ [1], whereas $\mathrm{NiCo}_{2} \mathrm{TeO}_{6}$ presents a twice higher value, thus nearing that of $\mathrm{Ni}_{2} \mathrm{MnTeO}_{6}$ [6]. However, one may only make a rough comparison between the static permittivity values, since they were measured at different frequencies: $44 \mathrm{kHz}, 0.1 \mathrm{MHz}$, and $1 \mathrm{MHz}$, for $\mathrm{Ni}_{3} \mathrm{TeO}_{6}, \mathrm{Ni}_{2} \mathrm{CoTeO}_{6} / \mathrm{NiCo}_{2} \mathrm{TeO}_{6}$, and $\mathrm{Ni}_{2} \mathrm{MnTeO}_{6}$, respectively.

The magnetic field dependences of magnetization of $\mathrm{Ni}_{2} \mathrm{CoTeO}_{6}$ and $\mathrm{NiCo}_{2} \mathrm{TeO}_{6}$ single crystals at $2 \mathrm{~K}$ and for $\boldsymbol{H}_{\text {ext }} \| a b$ axis are shown in Fig. 2(b). $\mathrm{Ni}_{2} \mathrm{CoTeO}_{6}$ presents a spin-flop transition at approximately $8 \mathrm{~T}$, similarly to $\mathrm{Ni}_{3} \mathrm{TeO}_{6}$ [1], however $\mathrm{NiCo}_{2} \mathrm{TeO}_{6}$ exhibits a dramatic decrease in the spin-flop critical field down to about $4 \mathrm{~T}$. Such a behavior can be explained by the helical spin structure (Fig. 1), and by the lack of a strong anisotropy direction of the FM layers in the $a b$ plane. The famous nonhysteretic behavior of $\mathrm{Ni}_{3} \mathrm{TeO}_{6}$ is absent in both compounds, which manifest a strong hysteresis in the magnetic field-dependent magnetization. The ceramic samples demonstrate similar magnetic phase transitions, albeit at slightly lower values of magnetic field, possibly due to grain boundary contributions (Fig. S3 in the SM) [14]. In addition, the hysteretic behavior becomes more pronounced for both samples on cooling (see Fig. S5.2).

\section{Spin and lattice excitations}

The family of the four Ni-based tellurates, $\mathrm{Ni}_{3} \mathrm{TeO}_{6}$, $\mathrm{Ni}_{2} \mathrm{MnTeO}_{6}, \mathrm{Ni}_{2} \mathrm{CoTeO}_{6}$ and $\mathrm{NiCo}_{2} \mathrm{TeO}_{6}$, crystalize in the polar $R 3$ space group, at least from room temperature (RT) down to $4 \mathrm{~K}$ and it is known that $\mathrm{Ni}_{3} \mathrm{TeO}_{6}$ keeps the same rhombohedral structure up to $1000 \mathrm{~K}$ [27]. As expected, all compounds present almost identical vibrational spectra [2,6,18]. All 18 vibrational modes predicted by factor group analysis [ $9 E\left(x, y, x^{2}-y^{2}, x y, x z, y z\right)$ and $\left.9 A\left(x^{2}+y^{2}, z^{2}, z\right)\right]$, both IR and Raman active, are present in the IR reflectivity spectra of both compounds [see Figs. 3(a) and 3(d)]. The corresponding mode frequencies are listed in Table S6 in the SM [14]. The anticipated weak temperature dependence of the IR reflectivity spectra of $\mathrm{Ni}_{2} \mathrm{CoTeO}_{6}$ and $\mathrm{NiCO}_{2} \mathrm{TeO}_{6}$ confirms the resemblance among the four compounds and the stability of the crystal structure in the whole temperature range studied. Naturally, the damping constants of the modes decrease on cooling. In analogy to the mode at $310 \mathrm{~cm}^{-1}$ in $\mathrm{Ni}_{3} \mathrm{TeO}_{6}$, the modes seen in $\mathrm{Ni}_{2} \mathrm{CoTeO}_{6}$ and $\mathrm{NiCo}_{2} \mathrm{TeO}_{6}$ at 224 and $211 \mathrm{~cm}^{-1}$, respectively, exhibit a conspicuous frequency hardening, accompanied by a decrease in damping. The observed phenomenon of mode intensity loss below $T_{\mathrm{N}}$ might be related to a coupling between the phonon and a lower energy magnon. As we show below, both compounds show several spin excitations in the $\mathrm{THz}$ range, thus a transfer of dielectric strength from the phonon to a spin excitation may take place when the magnetic ordering occurs and the magnon becomes electrically active (i.e., it contributes to the permittivity), which is a typical signature of electromagnons. 

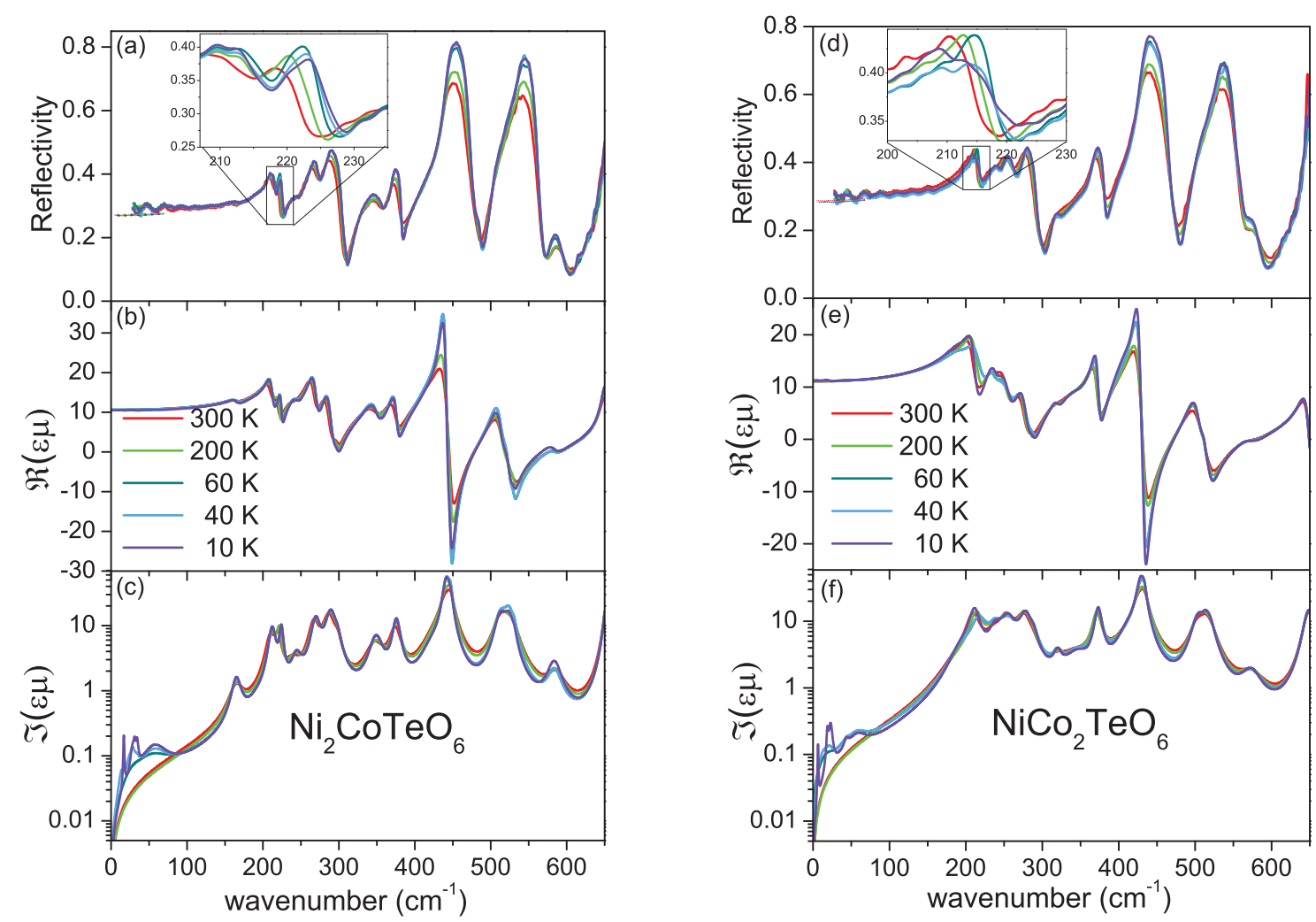

FIG. 3. Temperature dependences of IR reflectivity spectra for (a) $\mathrm{Ni}_{2} \mathrm{CoTeO}_{6}$ and (d) $\mathrm{NiCo}_{2} \mathrm{TeO}_{6}$ ceramics, at selected temperatures. The reflectivity spectra calculated using the $\mathrm{THz}$ range data are also shown, for frequencies below $70 \mathrm{~cm}^{-1}$, by dense dotted lines. Insets: Temperature dependences of IR reflectivity of the modes near (a) $220 \mathrm{~cm}^{-1}$ and (d) $215 \mathrm{~cm}^{-1}$, showing conspicuous decrease in damping on cooling, accompanied by frequency increase. (b), (e) Real and (c), (f) imaginary parts of the product of complex permittivity and complex magnetic permeability, as obtained from the fits.

The spectra were fit assuming a sum of independent harmonic oscillators, as mentioned in Sec. II. The products of the complex permittivity $\varepsilon$ and permeability $\mu$ spectra obtained by the fits for both compounds are plotted in Figs. 3(b), 3(c), 3(e), and 3(f). Although, the THz spectra are analyzed below in detail, here it is worth mentioning that the IR spectra were fit simultaneously with the $\mathrm{THz}$ spectra. In the low-frequency $\Im(\varepsilon \mu)$ spectra [Figs. 3(c) and 3(f)] one can notice weak spin excitations appearing below $T_{\mathrm{N}}$, as measured by the $\mathrm{THz}$ spectroscopy. Since we do not know whether they contribute only to permittivity or permeability, we present their product.

Note that these modes are very weak, therefore they are not visible in the real part of spectra. All the modes seen above $150 \mathrm{~cm}^{-1}$ are polar phonons, i.e., they contribute only to permittivity and $\mu^{\prime}=1$ in this range.

For both compounds a significant number of sharp modes can be seen in the temperature dependent Raman spectra of single crystals at all possible polarization configurations $[z(x y) \bar{z}, z(x x) \bar{z}, z(y y) \bar{z}, x(y z) \bar{x}, x(y y) \bar{x}, x(z z) \bar{x}]$ taken in the backscattering geometry. Here we present only $z(x y) \bar{z}$ spectra (Fig. 4), where the magnetic excitation is more pronounced comparing to the other configurations.

All above-mentioned configurations measured at $4 \mathrm{~K}$ are plotted in Fig. S6 in the SM [14]. The temperature dependence of $x(z y) \bar{x}, x(y y) \bar{x}$ and $x(z z) \bar{x}$ polarization configurations for both compounds are presented in the Figs. S6.1.2 and S6.1.3, together with a schematic representation of the configurations used and their respective Porto notations (Fig. S6.1) in the SM [14]. The Raman modes from all possible polarization configurations for $\mathrm{Ni}_{2} \mathrm{CoTeO}_{6}$ and $\mathrm{NiCo}_{2} \mathrm{TeO}_{6}$ are listed together with the IR modes in Table S6.2.1 in the SM [14]. Both compounds show an additional mode in the low-frequency range, at approximately $15-16 \mathrm{~cm}^{-1}$, which turns up below the AFM transition, therefore we assume it is of magnetic origin. This low frequency excitation appears in most polarization configurations, as seen in the SM [14], however, the strongest intensity corresponds to $z(x y) \bar{z}$, reflecting the $a b$-plane-sensitivity of the magnon. Note that the observed low-intensity modes observed in other polarized spectra may also originate in polarization leakage, due to slight sample misalignments. In addition, for $x(y z))^{\prime}$ in $\mathrm{Ni}_{2} \mathrm{CoTeO}_{6}$, a broader mode is seen at around $67 \mathrm{~cm}^{-1}$, which might be of two-magnon scattering origin. As we show below in more detail, both modes are also observed in the $\mathrm{THz}$ spectra.

The temperature dependence of the imaginary part of the index of refraction in the THz range is shown in Figs. 5(b) and 5(f) for $\mathrm{Ni}_{2} \mathrm{CoTeO}_{6}$ and $\mathrm{NiCo}_{2} \mathrm{TeO}_{6}$, respectively (ceramics of $\sim 1.9$-mm thickness). The real part of the index of refraction for both compounds is presented in Fig. S6.2 in the SM [14]. The spectra were measured from 300 down to $5 \mathrm{~K}$. An initial decrease in the real part of the index of refraction on cooling is observed, followed by the appearance of two modes below $T_{\mathrm{N}}$ for both $\mathrm{Ni}_{2} \mathrm{CoTeO}_{6}\left(T_{\mathrm{N}} \approx 54 \mathrm{~K}\right)$ and $\mathrm{NiCo}_{2} \mathrm{TeO}_{6}$ $\left(T_{\mathrm{N}} \approx 49 \mathrm{~K}\right)$. All excitations sharpen and harden on cooling, 

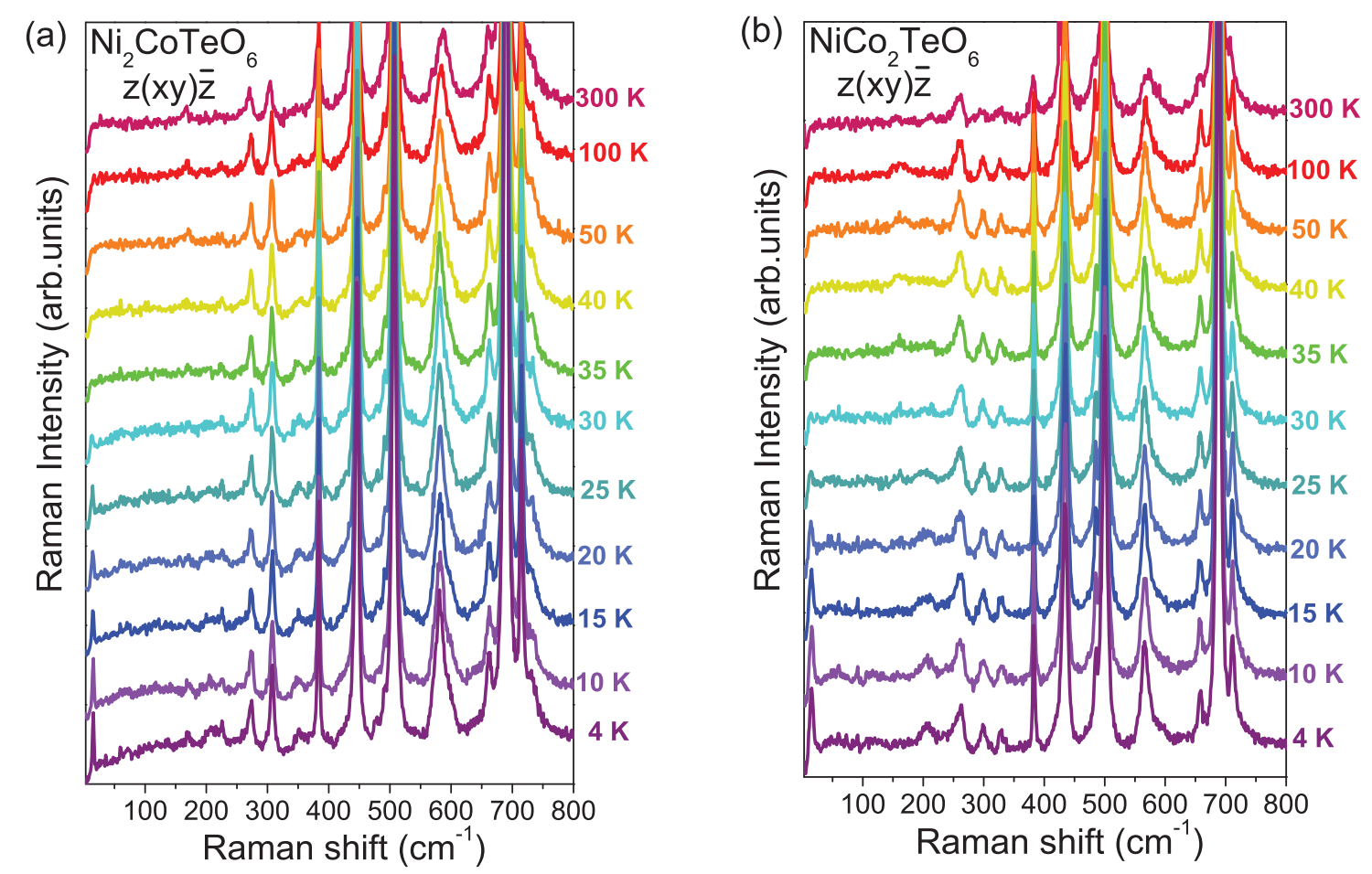

FIG. 4. Temperature dependences of Raman spectra for (a) $\mathrm{Ni}_{2} \mathrm{CoTeO}_{6}$ and (b) $\mathrm{NiCo}_{2} \mathrm{TeO}_{6}$ single crystals, measured at $z(x y) \bar{z}$ polarization configuration. A spin excitation appears below $T_{\mathrm{N}}$ at $\sim 15 \mathrm{~cm}^{-1}$ for both compounds.

with evident splitting for the broader ones. The spectra were fit together with those of IR reflectivity, and the corresponding frequencies, damping constants, and mode strengths are listed in Table S6.2.1 in the SM [14].

At the lowest temperature of $5 \mathrm{~K}$ the $\mathrm{THz}$ spectrum of $\mathrm{Ni}_{2} \mathrm{CoTeO}_{6}$ displays five modes, whereas $\mathrm{NiCo}_{2} \mathrm{TeO}_{6}$ presents six of them. The rapid strengthening of these modes upon cooling below $T_{\mathrm{N}}$, especially of the sharp ones, suggests that they correspond to spin excitations. As mentioned above, the $15 \mathrm{~cm}^{-1}$ mode is simultaneously seen in the Raman spectra of both compounds. Magnons are mostly very weak or inactive in Raman spectra. Nevertheless, in some magnetic materials like orthoferrites, the magnons are clearly observed in Raman spectra [30] and they are also IR active, despite their centrosymmetric Pnma crystal structure. $\mathrm{Ni}_{2} \mathrm{CoTeO}_{6}$ and $\mathrm{NiCo}_{2} \mathrm{TeO}_{6}$ have a noncentrosymetric $R 3$ crystal structure, so the phonons and magnons can be both IR and Raman active. Since the magnons were observed in the $z(x x) \bar{z}$ and $z(x y) \bar{z}$ Raman spectra, they belong to the $E$ symmetry. As the phonons near $210-220 \mathrm{~cm}^{-1}$ reduce their oscillator strengths on cooling below $T_{\mathrm{N}}$, they probably transfer their dielectric strengths to the strongest Raman-active magnons near $17 \mathrm{~cm}^{-1}$ and therefore we assume that these excitations can be called electromagnons. Note the small strengths of all our spin excitations in the THz spectra measured in transmission using a 1.9-mm-thick sample. The broader mode near $60 \mathrm{~cm}^{-1}$ (Fig. 3) is also seen in Raman spectra (Figs. S6.1.2 and S6.1.3), but it most probably corresponds to two-magnon scattering.

Spectra of the extinction coefficient obtained by timedomain $\mathrm{THz}$ measurements in the Voigt configuration with external magnetic field $\boldsymbol{H}_{\text {ext }} \perp \boldsymbol{E}^{\omega}$ up to $7 \mathrm{~T}$ and at temperatures of 30 and $5 \mathrm{~K}$ are presented in Figs. 5(c) and 5(d) and 5(g) and 5 (h) for each compound, respectively. The spectra of the real parts of the index of refraction are presented in Fig. S6.2.2 in the SM [14]. In both compounds, the magnons can be tuned by applying external magnetic field, upon which they exhibit noticeable peak sharpening and frequency shifts. The magnetic field dependences of the spin-wave frequencies at $5 \mathrm{~K}$, obtained by the fits, are summarized in Figs. 6(a) and 6(b). In $\mathrm{Ni}_{2} \mathrm{CoTeO}_{6}$, the sharp mode seen at $30 \mathrm{~K}$ around $17 \mathrm{~cm}^{-1}$ (0 T) [Fig. 5(c)] softens down to $14 \mathrm{~cm}^{-1}$ at $7 \mathrm{~T}$, whereas the higher-frequency modes near $30 \mathrm{~cm}^{-1}$ are less sensitive to $\boldsymbol{H}_{\text {ext }}$. The $17 \mathrm{~cm}^{-1}(0 \mathrm{~T})$ mode was also seen in Raman spectra, as mentioned above. The small discrepancy between the frequencies can be explained by the experimental error and it may also slightly vary in different experimental setups. The sharpening and softening of the latter mode is even more pronounced at $5 \mathrm{~K}$ [Fig. 5(d)].

The changes in the magnons response in external magnetic field are even more pronounced in $\mathrm{NiCo}_{2} \mathrm{TeO}_{6}$. At $30 \mathrm{~K}$ [Fig. 5(g)], the two strongest modes near 7 and $23 \mathrm{~cm}^{-1}$ at $0 \mathrm{~T}$, harden to 13 and $28 \mathrm{~cm}^{-1}$, respectively, with rising field up to $7 \mathrm{~T}$. At $5 \mathrm{~K}$ the spectra are much richer [Fig. 5(h)]. The magnon frequency shifts due to external magnetic field can be seen in Fig. 6(b). The marked discontinuities that occur at $\sim 4 \mathrm{~T}$ in the frequencies of at least four of the excitations, are probably linked to the spin-flop transition, observed also by the magnetization measurements [Fig. 2(b)]. Two new modes seem to appear at around $30 \mathrm{~cm}^{-1}$ above $3 \mathrm{~T}$. These new excitations might also correspond to a splitting of the neighboring singlet mode, triggered by the spin-flop transition. The convoluted noncollinear magnetic behavior of the Co-rich compounds was foreseen due to the intriguing 

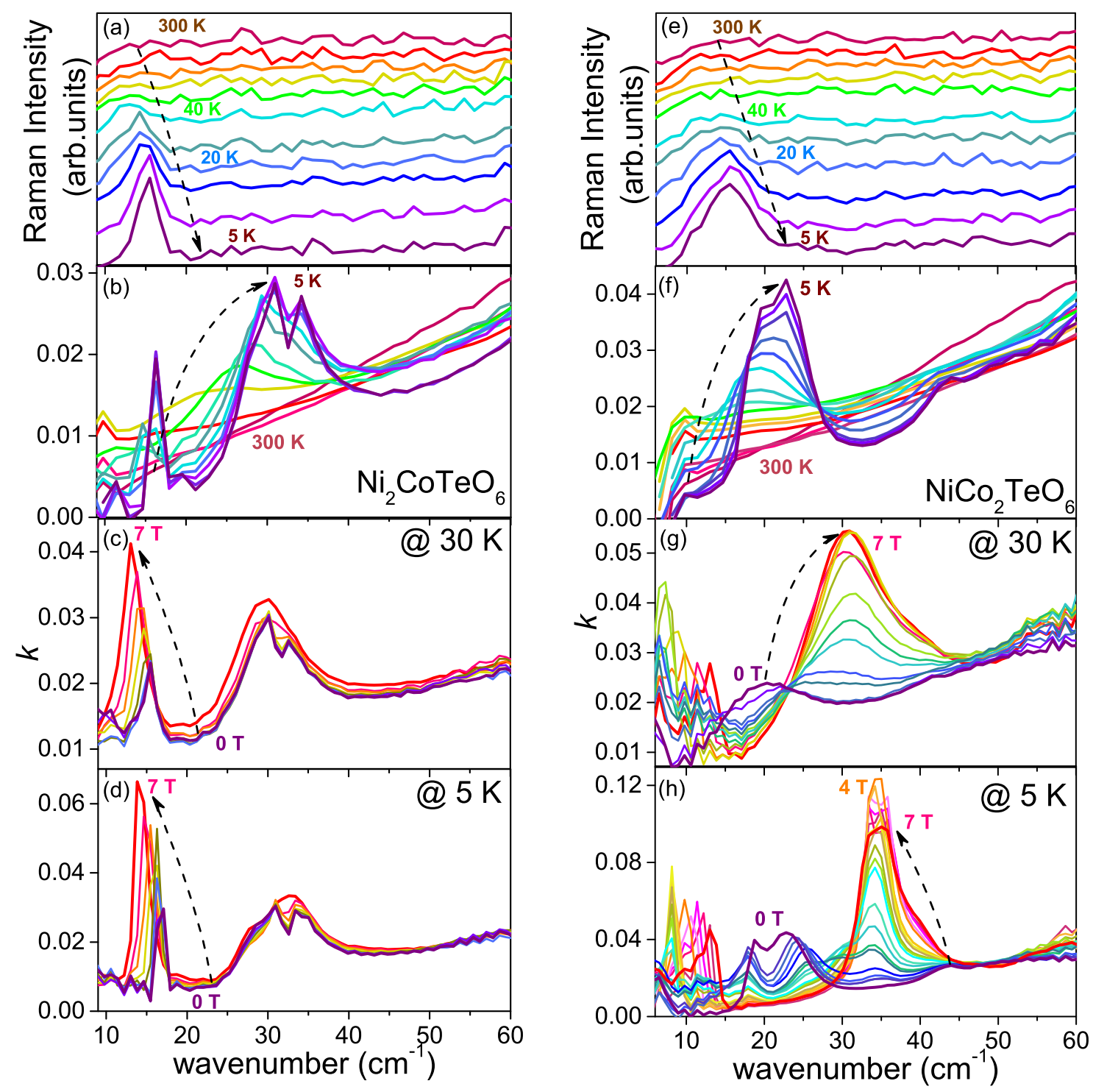

FIG. 5. (a),(e) Temperature dependences of the low frequency $z(x y) \bar{z}$ Raman spectra, (b),(f) temperature and (c),(d),(g),(h) magnetic field dependences of the imaginary part of the index of refraction from $\mathrm{THz}$ measurements for $\mathrm{Ni}_{2} \mathrm{CoTeO}_{6}$ (a)-(d) and $\mathrm{NiCo}_{2} \mathrm{TeO}_{6}(\mathrm{e})-(\mathrm{h})$. At least five spin excitations appear below $T_{\mathrm{N}}$ for both compounds, and the one at $\sim 15 \mathrm{~cm}^{-1}$ is simultaneously seen in the Raman spectra. The arrows in parts (a), (b), (e), and (f) indicate the temperature decrease from 300 to $5 \mathrm{~K}$, whereas in (c), (d), (g), and (h), they show the increase of the external magnetic field from 0 to $7 \mathrm{~T}$.

magnetism of $\mathrm{Co}_{3} \mathrm{TeO}_{6}[7,8,10]$, as well as the spin-orbit coupling contribution from $\mathrm{Co}^{3+}(3 d[6])$.

The $15-\mathrm{cm}^{-1}$ mode seen in Raman spectra [Fig. 5(e)], possibly corresponds to the one in the $\mathrm{THz}$ spectra at $\sim 17 \mathrm{~cm}^{-1}(0 \mathrm{~T})$, and it shows a strong magnetic field dependence. Since this mode (together with its higher-frequency component near $22 \mathrm{~cm}^{-1}$ ) increases its strength below $T_{\mathrm{N}}$ in THz spectra [Fig. 5(f)] and the phonon mode near $215 \mathrm{~cm}^{-1}$ reduces its dielectric strength on cooling below $T_{\mathrm{N}}$ (Fig. 3), these modes are probably coupled. It strongly suggests that the spin excitation seen near 17 and $22 \mathrm{~cm}^{-1}$ are electrically active and can be called electromagnons.

The discrepancies in the $T_{\mathrm{N}}$ values observed by Raman spectroscopy (the magnetic modes disappear already at $\sim 40 \mathrm{~K}$, more than $10 \mathrm{~K}$ below the AFM phase transition) could be explained by thermo-lag effects, laser-heating, and/or random crystallite orientation in the polycrystalline samples.
Note that the observed excitations cannot correspond to crystal field splitting caused by the octahedral coordination geometry in $\mathrm{Ni}, \mathrm{Co}$, and $\mathrm{Te}$, since the $3 d$-related crystal field splitting values lie in the UV and visible range [34], whereas the $4 f$-related splitting reaches down to the far-IR range [35].

Substitution of $\mathrm{Ni}$ by $\mathrm{Mn}$ and $\mathrm{Co}$ in the $\mathrm{Ni}_{3-x}(\mathrm{Co}, \mathrm{Mn})_{x} \mathrm{TeO}_{6}(x=0-2)$ compound family preserves the polar $R 3$ crystal structure. However, the different magnetic anisotropies introduced by the magnetic cations $\mathrm{Ni}(\mathrm{II})(3 d$ [8]), $\mathrm{Mn}(\mathrm{II})(3 d$ [5]) and $\mathrm{Co}(\mathrm{II})(3 d$ [7]) dramatically affect the magnetic phase transitions and the spin order (see Table I). The differences in the spin modulations of the compounds are reflected in the THz spectra (Fig. 7). Very recent neutron studies of the magnetic ordering of $\mathrm{Ni}_{3} \mathrm{TeO}_{6}$ single crystals have revealed that the commensurately modulated collinear antiferromagnetic structure changes above $8.6 \mathrm{~T}$ to an incommensurately modulated conical spiral 

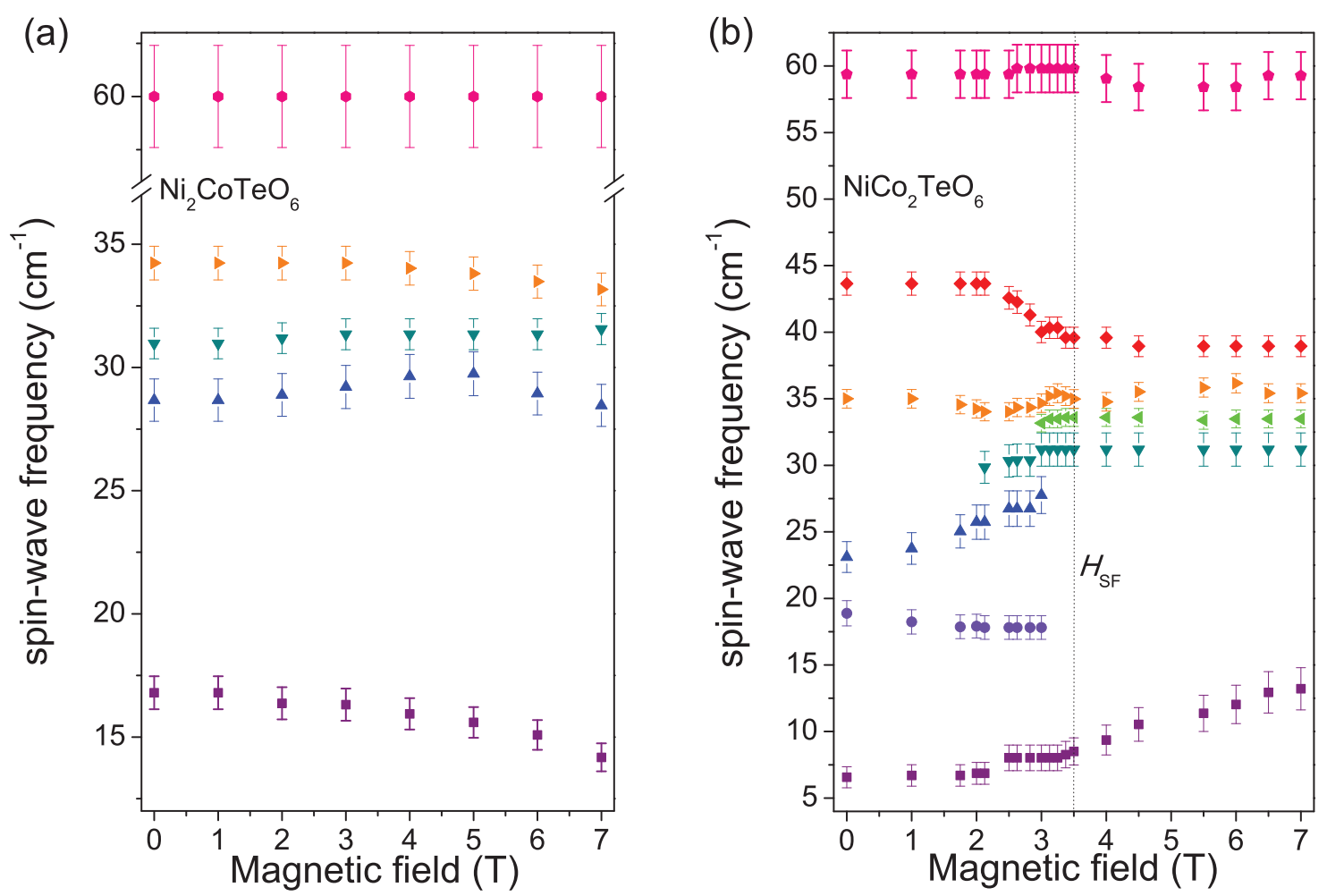

FIG. 6. Magnetic field dependence of spin-wave frequencies for (a) $\mathrm{Ni}_{2} \mathrm{CoTeO}_{6}$ and (b) $\mathrm{NiCo}_{2} \mathrm{TeO}_{6}$, at $5 \mathrm{~K}$. In the latter, the spin-flop transition is clearly seen at approximately $3 \mathrm{~T}$ (designated by the violet stripe), by the steps on the magnon branches, as well as both the appearance of new modes and extinction of ones present in the low-field phase.

one with significant spin components in the $a b$ plane [33]. One can expect that the $\mathrm{THz}$ spectra of $\mathrm{Ni}_{3} \mathrm{TeO}_{6}$ taken above 8.6 $\mathrm{T}$ should resemble the spectra of $\mathrm{NiCo}_{2} \mathrm{TeO}_{6}$ at zero magnetic field. Unfortunately, the magnetic cryostat used for measuring the $\mathrm{THz}$ spectra operates up to $7 \mathrm{~T}$. In addition, the absence of evidence of the exact spin order at external magnetic field for all compounds (except for $\mathrm{Ni}_{3} \mathrm{TeO}_{6}$ ) and the fact that the $\mathrm{THz}$ experiments were performed with polycrystalline samples prevents us from drawing a detailed comparison between compounds.

Large enough single crystals are required for more extensive $\mathrm{THz}$ measurements, in order to resolve the relation between crystal symmetry and magnon symmetry and propagation. Also the alternative of studying directional dichroism is currently not feasible due to the small size of the available crystals, and because the pyroelectric polarization is most likely nonswitchable [36]. Finally, single crystals could also serve for performing pyrocurrent measurements in external magnetic field, in order to examine the static ME coupling, and compare it with the colossal $\mathrm{ME}$ effect observed in $\mathrm{Ni}_{3} \mathrm{TeO}_{6}$.

\section{CONCLUSIONS}

New compounds from the family of Ni-based tellurates, $\mathrm{Ni}_{2} \mathrm{CoTeO}_{6}$ and $\mathrm{NiCo}_{2} \mathrm{TeO}_{6}$, were successfully synthesized in the form of single crystals and ceramics. Co substitution of $\mathrm{Ni}$ in $\mathrm{Ni}_{3} \mathrm{TeO}_{6}$ preserves the $\mathrm{ME}$ properties of the parent structure, and it enhances the dynamical ME coupling. The noncentrosymmetric rhombohedral $R 3$ structure is maintained at least up to RT for both $\mathrm{Ni}_{2} \mathrm{CoTeO}_{6}$ and $\mathrm{NiCo}_{2} \mathrm{TeO}_{6}$, even though $\mathrm{Co}_{3} \mathrm{TeO}_{6}$ has a monoclinic nonpolar structure with the space group $C 2 / c$. Unfortunately, evidence of ferroelectricity is still missing, since our single-crystalline samples were too

TABLE I. Overview of main characteristics of magnetic phase transitions and spin orders for the $\mathrm{Ni}_{3-x}\left(\mathrm{Co}, \mathrm{Mn}_{x} \mathrm{TeO}_{6}(x=0-2)\right.$ compounds: paramagnetic to AFM phase transition at $T_{\mathrm{N}}$, spin-flop transition at external magnetic field $H_{\mathrm{SF}}$, type of spin order and spin orientation, and spin order propagation vector $k$. The metamagnetic phase transitions observed in $\mathrm{Ni}_{3} \mathrm{TeO}_{6}$ at higher magnetic fields (up to 92 T) [31-33] are not included here.

\begin{tabular}{lcccc}
\hline \hline & $\mathrm{Ni}_{3} \mathrm{TeO}_{6}[1,3]$ & $\mathrm{Ni}_{2} \mathrm{MnTeO}_{6}[6]$ & $\mathrm{Ni}_{2} \mathrm{CoTeO}_{6}$ & $\mathrm{NiCo}_{2} \mathrm{TeO}_{6}$ \\
\hline$T_{\mathrm{N}}(\mathrm{K})$ & 53 & 70 & 55 & 52 \\
$H_{\mathrm{SF}}(\mathrm{T})$ & 8.2 & 4.5 & 8 & 4 \\
Spin order & collinear $c$ axis & collinear $c$ axis & helical $a b$ plane & helical $a b$ plane \\
$\boldsymbol{k}$ & {$[0,0,0.5]$} & {$[0,0,0.5]$} & {$[0,0,1.299(4)]$} & {$[0,0,1.2109(1)]$} \\
\hline \hline
\end{tabular}




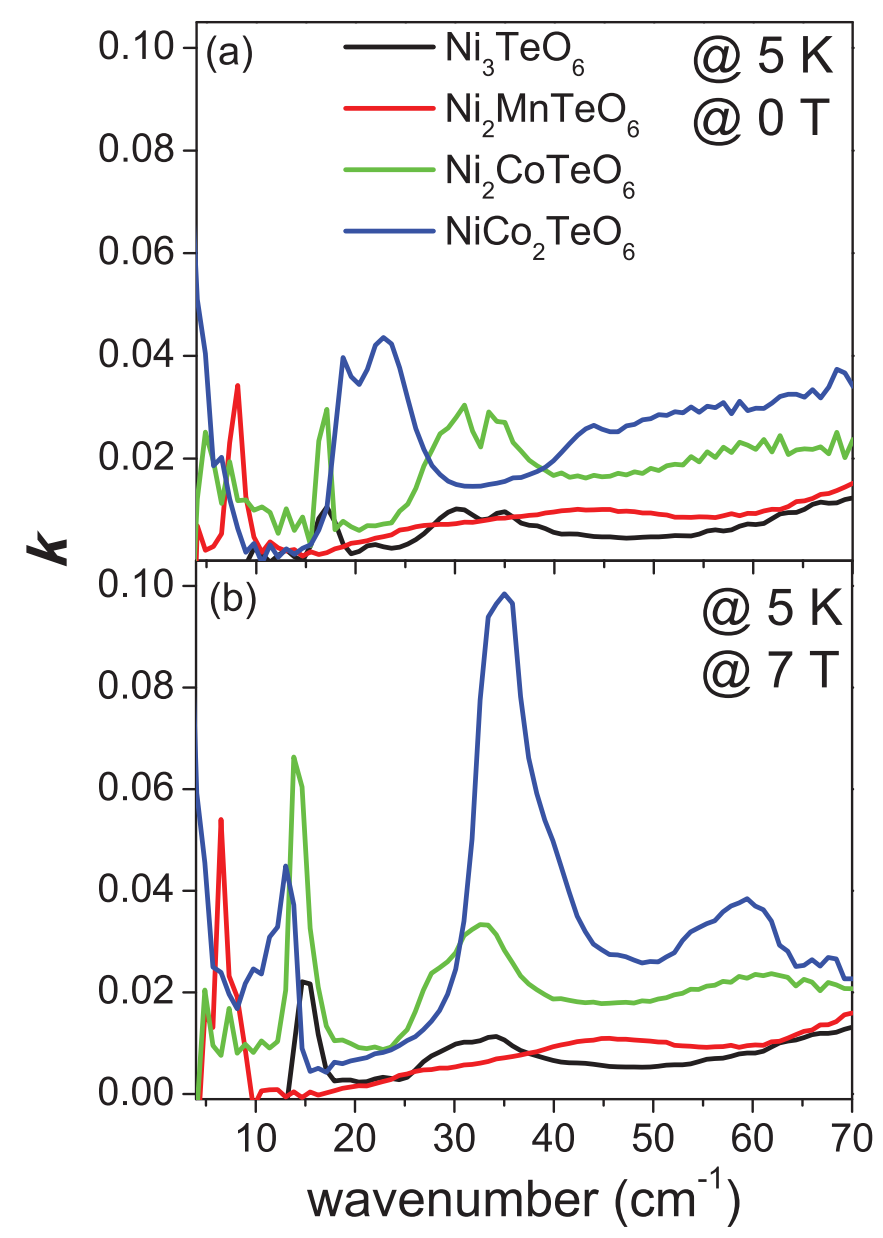

FIG. 7. Comparison of $\mathrm{THz}$ extinction coefficient spectra $k$ for $\mathrm{Ni}_{3-x}(\mathrm{Co}, \mathrm{Mn})_{x} \mathrm{TeO}_{6}(x=0,1,2)$ compounds taken at $5 \mathrm{~K}$ at (a) zero magnetic field and (b) $7 \mathrm{~T}$.

small for pyrocurrent or ferroelectric hysteresis loops measurements. The magnetic ordering appears in single crystals (ceramics) at $T_{\mathrm{N}}=55(54)$ and $52(49) \mathrm{K}$ for $\mathrm{Ni}_{2} \mathrm{CoTeO}_{6}$ and $\mathrm{NiCo}_{2} \mathrm{TeO}_{6}$, respectively.
In contrast to the magnetic ordering in $\mathrm{Ni}_{3} \mathrm{TeO}_{6}$ and $\mathrm{Ni}_{2} \mathrm{MnTeO}_{6}$, which are collinear along the $c$ axis, the two compounds under study present a helical noncollinear structure with the spins in the $a b$ plane. Hysteretic spin-flop transitions were observed in both compounds, near $8 \mathrm{~T}$ $\left(\mathrm{Ni}_{2} \mathrm{CoTeO}_{6}\right)$ and $4 \mathrm{~T}\left(\mathrm{NiCo}_{2} \mathrm{TeO}_{6}\right)$, in contrast to $\mathrm{Ni}_{3} \mathrm{TeO}_{6}$ and $\mathrm{Ni}_{2} \mathrm{MnTeO}_{6}$, which did not show any hysteretic behavior.

Below $T_{\mathrm{N}}$ in the $\mathrm{THz}$ range both compounds demonstrate numerous spin excitations, which are markedly influenced by external magnetic field, with clear modifications at the spin-flop transition of $\sim 4 \mathrm{~T}$ for the case of $\mathrm{NiCo}_{2} \mathrm{TeO}_{6}$. The Co-richer compound $\mathrm{NiCo}_{2} \mathrm{TeO}_{6}$ prevails, manifesting six magnons unusually strongly dependent on magnetic field. Both compounds present at least one magnetic excitation simultaneously seen by Raman spectroscopy, thus they can be assigned to electromagnons.

\section{ACKNOWLEDGMENTS}

The work of M.G., Z.D., and M.R. was supported by the NSF-DMR, Grant No. 1507252. M.R. also acknowledges the Juan de la Cierva program of the Spanish Ministry of Economy and Competitiveness for Grant No. FPDI-2013-17582. The work of S.K., S.S., F.B., C.K., and F.K. was supported by the Czech Science Foundation (Project No. 18-09265S) and by Operational Programme Research, Development and Education financed by European Structural and Investment Funds and the Czech Ministry of Education, Youth and Sports (Project No. SOLID21 - Z.02.1.01/0.0/0.0/16_019/0000760). This project has received funding from the European Union's Horizon 2020 research and innovation programme under the Marie Sklodowska-Curie Grant Agreement No. 838220. D.L. and S.S. acknowledge the European Regional Development Fund in the IT4Innovations national supercomputing centre-Path to Exascale (Project No. CZ.02.1.01/0.0/0.0/16_013/0001791) within the Operational Programme Research, Development, and Education, and D.L. acknowledges also Project No. 17-27790S of the Czech Science Foundation. The authors acknowledge the Science and Technology Facility Council (STFC, UK) for the provision of neutron beam time on the WISH diffractometer.
[1] Y. S. Oh, S. Artyukhin, J. J. Yang, V. Zapf, J. W. Kim, D. Vanderbilt, and S.-W. Cheong, Nat. Commun. 5, 3201 (2014).

[2] S. Skiadopoulou, F. Borodavka, C. Kadlec, F. Kadlec, M. Retuerto, Z. Deng, M. Greenblatt, and S. Kamba, Phys. Rev. B 95, 184435 (2017).

[3] I. Živković, K. Prša, O. Zaharko, and H. Berger, J. Phys. Condens. Matter 22, 56002 (2010).

[4] V. G. Bar'yakhtar and I. E. Chupis, Sov. Phys. Solid State 11, 2628 (1970).

[5] A. Pimenov, A. A. Mukhin, V. Y. Ivanov, V. D. Travkin, A. M. Balbashov, and A. Loidl, Nat. Phys. 2, 97 (2006).

[6] M. Retuerto, S. Skiadopoulou, F. Borodavka, C. Kadlec, F. Kadlec, J. Prokleška, Z. Deng, J. A. Alonso, M. T. FernandezDiaz, F. O. Saouma, J. I. Jang, D. Legut, S. Kamba, and M. Greenblatt, Phys. Rev. B 97, 144418 (2018).
[7] S. A. Ivanov, R. Tellgren, C. Ritter, P. Nordblad, R. Mathieu, G. Andre, N. V. Golubko, E. D. Politova, and M. Weil, Mater. Res. Bull. 47, 63 (2012).

[8] M. Hudl, R. Mathieu, S. A. Ivanov, M. Weil, V. Carolus, T. Lottermoser, M. Fiebig, Y. Tokunaga, Y. Taguchi, Y. Tokura, and P. Nordblad, Phys. Rev. B 84, 180404 (2011).

[9] W.-H. Li, C.-W. Wang, D. Hsu, C.-H. Lee, C.-M. Wu, C.-C. Chou, H.-D. Yang, Y. Zhao, S. Chang, J. W. Lynn, and H. Berger, Phys. Rev. B 85, 094431 (2012).

[10] P. Tolédano, V. Carolus, M. Hudl, T. Lottermoser, D. D. Khalyavin, S. A. Ivanov, and M. Fiebig, Phys. Rev. B 85, 214439 (2012).

[11] A. B. Harris, Phys. Rev. B 85, 100403 (2012).

[12] C.-W. Wang, C.-H. Lee, C.-Y. Li, C.-M. Wu, W.-H. Li, C.-C. Chou, H.-D. Yang, J. W. Lynn, Q. Huang, A. B. Harris, and H. Berger, Phys. Rev. B 88, 184427 (2013). 
[13] H. Singh, H. Ghosh, T. V. Chandrasekhar Rao, G. Sharma, J. Saha, and S. Patnaik, J. Appl. Phys. 119, 044104 (2016).

[14] See Supplemental Material at http://link.aps.org/supplemental/ 10.1103/PhysRevB.101.014429 for crystal and magnetic structures (XRD and PND data), magnetic studies, additional Raman and THz spectra, table of modes seen in IR and Raman spectra.

[15] G. Kresse and J. Furthmüller, Phys. Rev. B 54, 11169 (1996).

[16] J. P. Perdew, K. Burke, and M. Ernzerhof, Phys. Rev. Lett. 77, 3865 (1996).

[17] A. I. Liechtenstein, V. I. Anisimov, and J. Zaanen, Phys. Rev. B 52, R5467 (1995).

[18] M. O. Yokosuk, A. al-Wahish, S. Artyukhin, K. R. O’Neal, D. Mazumdar, P. Chen, J. Yang, Y. S. Oh, S. A. McGill, K. Haule, S.-W. Cheong, D. Vanderbilt, and J. L. Musfeldt, Phys. Rev. Lett. 117, 147402 (2016).

[19] M. Rössle, C. N. Wang, P. Marsik, M. Yazdi-Rizi, K. W. Kim, A. Dubroka, I. Marozau, C. W. Schneider, J. Humlíček, D. Baeriswyl, and C. Bernhard, Phys. Rev. B 88, 104110 (2013).

[20] L. C. Chapon, P. Manuel, P. G. Radaelli, C. Benson, L. Perrott, S. Ansell, N. J. Rhodes, D. Raspino, D. Duxbury, E. Spill, and J. Norris, Neutron News 22, 22 (2011).

[21] A. Coelho, Topas Academic: General Profile and Structure Analysis Software for Powder Diffraction Data (Bruker AXS, Karlsruhe, Germany, 2012).

[22] V. Petříček, M. Dušek, and L. Palatinus, Z. Kristallogr. Cryst. Mater. 229, 345 (2014).

[23] H. T. Stokes, D. M. Hatch, and B. J. Campbell, ISOTROPY Software Suite, Iso.Byu.Edu (2019).

[24] B. J. Campbell, H. T. Stokes, D. E. Tanner, and D. M. J. Hatch, J. Appl. Crystallogr. 39, 607 (2006).

[25] F. Gervais, High-Temperature Infrared Reflectivity Spectroscopy by Scanning Interface, in Infrared Millimeter Waves,
Vol. 8, edited by K. J. Button (Academic, New York, 1983), Chap. 7, p. 279.

[26] M. Tyunina, A. Dejneka, D. Rytz, I. Gregora, F. Borodavka, M. Vondracek, and J. Honolka, J. Phys. Condens. Matter 26, 125901 (2014).

[27] S. A. Ivanov, R. Mathieu, P. Nordblad, R. Tellgren, C. Ritter, E. Politova, G. Kaleva, A. Mosunov, S. Stefanovich, and M. Weil, Chem. Mater. 25, 935 (2013).

[28] K. L. Ji, E. Solana-Madruga, A. M. Arevalo-Lopez, P. Manuel, C. Ritter, A. Senyshyn, and J. P. Attfield, Chem. Commun. 54, 12523 (2018).

[29] Á. M. Arévalo-López, E. Solana-Madruga, E. P. ArévaloLópez, D. Khalyavin, M. Kepa, A. J. Dos Santos-García, R. Sáez-Puche, and J. P. Attfield, Phys. Rev. B 98, 214403 (2018).

[30] R. M. White, R. J. Nemanich, and C. Herring, Phys. Rev. B 25, 1822 (1982).

[31] J. W. Kim, S. Artyukhin, E. D. Mun, M. Jaime, N. Harrison, A. Hansen, J. J. Yang, Y. S. Oh, D. Vanderbilt, V. S. Zapf, and S.-W. Cheong, Phys. Rev. Lett. 115, 137201 (2015).

[32] M. O. Yokosuk, S. Artyukhin, A. Al-Wahish, X. Wang, J. Yang, Z. Li, S.-W. Cheong, D. Vanderbilt, and J. L. Musfeldt, Phys. Rev. B 92, 144305 (2015).

[33] J. Lass, C. Røhl Andersen, H. K. Leerberg, S. Birkemose, S. Toth, U. Stuhr, M. Bartkowiak, C. Niedermayer, Z. Lu, R. Toft-Petersen, M. Retuerto, J. Okkels Birk, and K. Lefmann, arXiv:1909.13734v2 (2019).

[34] M. E. Foglio and G. E. Barberis, Brazilian J. Phys. 36, 40 (2006).

[35] L. Chaix, S. de Brion, S. Petit, R. Ballou, L.-P. Regnault, J. Ollivier, J.-B. Brubach, P. Roy, J. Debray, P. Lejay, A. Cano, E. Ressouche, and V. Simonet, Phys. Rev. Lett. 112, 137201 (2014).

[36] X. Wang, F.-T. Huang, J. Yang, Y. S. Oh, and S.-W. Cheong, APL Mater. 3, 076105 (2015). 